\title{
Aplicabilidad del método de los elementos finitos en el análisis y dimensionamiento de losas JCPC para carreteras de dos carriles
}

\author{
Applicability of the finite element method in analysis and \\ sizing of JCPC slabs for two-lane roads
}

\author{
Myriam Rocío Pallares-Muñoz \\ M. Sc. Métodos Numéricos en Ingeniería \\ Universidad Surcolombiana \\ Neiva, Colombia \\ myriam.pallares@usco.edu.co
}

\author{
Julián Andrés Pulecio-Díaz \\ M. Sc. Construcción Obras Viales \\ Universidad Surcolombiana \\ Neiva, Colombia \\ julian.pulecio@usco.edu.co
}

\begin{abstract}
Resumen-Se analizaron y dimensionaron losas planas de concreto con juntas (Jointed-Plain-Concrete-Pavement "JCPC" en inglés) para carreteras de dos carriles por medio del modelador de elementos finitos EverFE 2.25, intentando reproducir de una manera más ajustada a la realidad los esfuerzos del conjunto de losas de pavimento. Para la validación se empleó el método analítico Westergaard 1948. Los resultados de esfuerzos y número de repeticiones de carga permitidos llevaron a concluir que el diseño de losas JCPC para carreteras de dos carriles no requiere el peso propio de la estructura en el análisis y dimensionamiento porque el porcentaje de variación obtenido es poco significativo. Por lo tanto, se recomienda omitir el parámetro densidad en el análisis para generar un coste computacional menor. Este estudio traza una ruta para seguir analizando otros factores que puedan afectar el dimensionamiento de pavimentos rígidos. Se espera en próximas investigaciones integrar cargas de distintos vehículos y los comportamientos producidos por la presencia de dovelas y barras de amarre en juntas transversales y longitudinales.
\end{abstract}

Palabras clave- Carreteras de dos carriles, EverFE 2.25, losas JCPC, modelado tridimensional de pavimentos, modelado de pavimentos con software libre, Westergaard 1948.

Abstract- Two-lane road JCPC (Jointed-Plain-Concrete-Pavement) slabs were analyzed and dimensioned using the EverFE 2.25 finite element modeler in order to reproduce the efforts of the pavement slab in a more realistic way. The "Westergaard"-1948 analytical method was used for the validation. With the results of efforts and the number of allowed repetitions of load we can conclude that the designs of JCPC slabs for two lanes do not require the analysis and dimensioning with own weight of the structure, because the percentage of variation is little significant. Therefore, we recommend omitting the density parameter in the analysis to generate a lower computational cost. This study shows us a route to continue analyzing other factors that influence the sizing of rigid pavements. We hope in future investigations to integrate loads of different vehicles and the behaviors produced by the presence of segments and tie bars in transverse and longitudinal joints.

Keywords- Two-lane roads, EverFE 2.25, Slabs JCPC, three-dimensional modeling of pavements, freeware pavement modeling, Westergaard 1948.

\section{INTRODUCCIÓN}

En la actualidad para el dimensionamiento de estructuras de pavimentos rígidos para carreteras de dos carriles se emplean métodos empíricos y empíricos-mecanísticos, definiendo comúnmente para ello: el eje de diseño (eje simple de 8.2T $(80 \mathrm{kN})$ ) o el espectro de carga, el espesor de las capas y las características de los materiales. De manera que se pueda calcular los máximos esfuerzos principales a tensión en las fibras inferiores o superiores de la losa (fatiga) [1]-[3] y deformaciones verticales en las capas granulares y subrasante (deformación permanente). Orientado a estudios en una a dos dimensiones que conllevan omitir condiciones de frontera más amplias. 
Este hecho genera la necesidad de realizar un análisis y dimensionamiento mucho más recíproco con la realidad, teniendo en cuenta un modelado en tercera dimensión, con y sin peso propio de la estructura, carga más agresiva y condiciones de liga entre losas y demás capas (base y subrasante). Para este propósito se empleó el modelador tridimensional de elementos finitos EverFE 2.25 y el método analítico Westergaard 1948 para validar los resultados.

\section{MÉTODOS}

El análisis se abordó por medio del modelador computacional de elementos finitos EverFE 2.25, validando sus resultados con el método analítico Westergaard 1948. La estructura de pavimento rígido está compuesta por una base granular y una capa de rodadura de losas JCPC (Jointed Plain Concrete Pavement) como se muestra en la Fig. 1.

Fig. 1. ESTRUCTURA PAVIMENTO RÍGIDO DE LOSAS JCPC

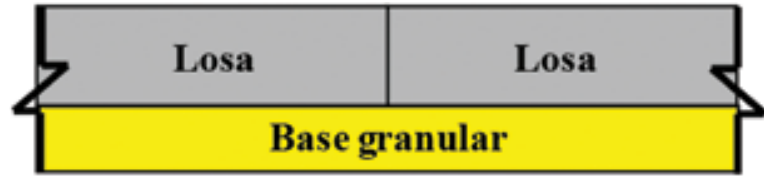

Subrasante

Fuente: los autores.

Las dimensiones de las capas de la estructura de pavimento modelado se presentan en la Tabla I. La Tabla I presenta los espesores en milímetros $(\mathrm{mm})$ y pulgadas (in), ya que el modelador de elementos finitos EverFE 2.25 y el método analítico de Westergaard 1948 requieren de este tipo de unidades. La condición de frontera considerada para la subrasante es infinita y como es común en el dimensionamiento de este tipo de estructuras se trabajaron las interfaces ligada entre subrasante y base granular y no ligada entre base granular y losas. Las losas son cuadradas de $3.5 \mathrm{~m} * 3.5 \mathrm{~m}$ y las aberturas de las juntas de $5 \mathrm{~mm}$ de espesor, ambos son parámetros geométricos que se anticipan a las fallas por contracción y alabeo del concreto hidráulico. Las fallas por contracción ocurren por secado y enfriado del material. Se dan usualmente entre las primeras 12 o 24 horas de ser colocado y colado el concreto hidráulico de cemento tipo I. Las fallas por alabeo se dan en el tiempo Jeong [4].
TABLA I

VALORES TÍPICOS DE ESPESORES DE CAPA DE UNA ESTRUCTURA DE PAVIMENTO RÍGIDO DE LOSAS JCPC

\begin{tabular}{|c|c|c|}
\hline Capa & Espesor $(\mathrm{mm})$ & Espesor (in) \\
\hline Losas JCPC & 228.6 & 9 \\
\hline Base granular & 150 & 5.905 \\
\hline Subrasante & infinita & infinita \\
\hline
\end{tabular}

Fuente: los autores.

Los módulos de rigidez tomados como referencia para cada una de las capas de acuerdo con las condiciones de trabajo del pavimento se muestran en la Tabla II. El módulo de reacción de la subrasante con denominación en inglés dense liquid subgrade, se determina por medio de un ensayo de placa de carga (PLT) corregido en ocasiones por: a) saturación del suelo cuando es cohesivo, b) asentamiento deficiente de la placa, comportamiento carga deformación no lineal o falla por corte, presentes al inicio del ensayo con un comportamiento variable en la gráfica carga versus deflexión, c) flexión de la placa soportante (cuando las deflexiones registradas mediante LVDTs o diales ubicados perimetralmente en la placa son menores a las del centro) Mallick y El-Korchi [5]-[7]. El módulo elástico de la base granular conocido como resiliente se determina por medio de ensayos triaxiales cíclicos Ping et al. [8], [9], que para el caso es un valor característico del módulo de reacción de la subrasante y el espesor de base granular empleados. El módulo elástico de las losas de concreto hidráulico se establece a través de ensayos estáticos a carga uniaxial Joh et al. [10], [11].

TABLA II

VALORES TÍPICOS DE MÓDULOS DE RIGIDEZ DE LAS CAPAS

\begin{tabular}{|c|c|c|c|c|}
\hline Capa & $\begin{array}{c}\text { Módulo } \\
\text { elástico } \\
(\mathrm{MPa})\end{array}$ & $\begin{array}{c}\text { Módulo } \\
\text { elástico } \\
\left(\mathrm{lb} / \text { pul }^{2}\right)\end{array}$ & $\begin{array}{c}\text { Módulo } \\
\text { reacción } \\
(\mathrm{MPa} / \mathrm{mm})\end{array}$ & $\begin{array}{c}\text { Módulo } \\
\text { reacción } \\
\left(\mathrm{lb} / \mathrm{pul}^{3}\right)\end{array}$ \\
\hline $\begin{array}{c}\text { Losas } \\
\text { JCPC }\end{array}$ & 28000 & 4000000 & - & - \\
\hline $\begin{array}{c}\text { Base } \\
\text { granular }\end{array}$ & 82.48 & 11782.85 & - & - \\
\hline $\begin{array}{c}\text { Subra- } \\
\text { sante }\end{array}$ & - & - & $0.04082 *$ & $150 *$ \\
\hline
\end{tabular}

Ya que la variación del módulo de Poisson no tiene incidencia considerable en el comportamiento del 
pavimento Huang [12], se emplearon los valores característicos de la tabla III.

TABLA III

VALORES TÍPICOS DE MÓDULO DE POISSON DE LAS CAPAS

\begin{tabular}{|c|c|}
\hline Capa & Módulo Poisson \\
\hline Losas JCPC & 0.15 \\
\hline Base granular & 0.40 \\
\hline Subrasante & - \\
\hline
\end{tabular}

Fuente: los autores.

La determinación de los parámetros de diseño de pavimentos rígidos tiene principio en el diseño empirico-mecanístico, que consiste en modelar la estructura a partir de la definición de los espesores y las rigideces (módulos elásticos, reacción y de Poisson) de cada capa para calcular las tensiones y compresiones provocadas por una carga tipo e identificar: a) el máximo esfuerzo principal a tensión en las fibras inferiores o superiores de la losa $\left(\sigma_{t}\right)$ capaz de producir rotura en las losas; este esfuerzo se compara con el límite admisible del concreto hidráulico $\left(\sigma_{\text {tadm }}\right)$ que es función del tránsito, esfuerzo a tensión aplicado y módulo de rotura, siendo este último parámetro determinado mediante el ensayo de viga de cuatro puntos; $b$ ) máximas deformaciones a compresión $\left(\varepsilon_{c}\right)$ en las capas granulares y subrasante que se compara con la admisible $\left(\varepsilon_{\text {cadm }}\right)$ y que depende del nivel de tránsito LCPC [13].

\subsection{Método analítico Westergaard 1948}

Se calcularon los máximos esfuerzos principales a tensión en el interior, esquina y borde de la losa, a partir de las expresiones (1), (2), (3), (4) y (5) de Westergaard 1948, introduciendo una presión de inflado distribuida uniformemente sobre un área circular de radio $0.2286 \mathrm{~m}$ (9 in) bajo una solicitación de carga de 40.1 kN (9000lb), que representa la mitad de un eje simple de rueda sencilla. El análisis permite identificar los esfuerzos característicos de control del diseño estructural del pavimento rígido, tales como los esfuerzos a tensión en las fibras inferiores o superiores de la losa (fatiga) Papagiannakis y Masad [14] Reddy [15].

$$
\ell=\left[\frac{E^{*} d^{3}}{12 *\left(1-\mu^{2}\right) * K}\right]^{0.25}=35.88 p u l
$$

$$
\begin{gathered}
b=\sqrt{1.6 * a^{2}+d^{2}}-0.675 * d=5.697 p u l \Rightarrow \\
a<1.724 * d=5.697 p u l<15.516 p u l \quad(2) \\
\sigma_{1}=\frac{3 * P *(1+\mu)}{d^{2} * 2 * \pi} *\left[\operatorname{In}\left(\frac{\ell}{b}\right)+0.6159\right]= \\
149.84 l b / p u l^{2}=1.05 M p a \text { en el int erior } \quad(3) \\
\sigma_{E}=\frac{3 * P}{d^{2}} *\left[1-\left(\frac{a^{*} \sqrt{2}}{\ell}\right)^{0.6}\right]= \\
193.0 l b / p u l^{2}=1.35 M p a \text { en la esquina } \\
\sigma_{B}=\frac{0.803 * P}{d^{2}} * \\
{\left[4 * \log \left(\frac{\ell}{a}\right)+0.666 * \frac{a}{\ell}-0.034\right]=} \\
284.10 l b / p u l^{2}= \\
1.99 M p a \text { en el borde }
\end{gathered}
$$

Donde $\mathrm{E}$ es el módulo elástico del concreto hidráulico, d espesor de la losa de concreto hidráulico, $\mu$ módulo de poisson del concreto hidráulico, $K$ módulo de reacción de la subrasante, $\mathrm{P}$ solicitación de carga, a radio del área circular, $\ell$ radio de rigidez relativa, $\sigma$ máximo esfuerzo principal a tensión en el interior de la losa, $\sigma_{E}$ máximo esfuerzo principal a tensión en la esquina de la losa y $\sigma_{B}$ máximo esfuerzo principal a tensión en el borde de la losa.

\subsection{Modelos de elementos finitos EverFE 2.25}

Los modelos de elementos finitos con el modelador tridimensional elástico EverFE 2.25 fueron validados con el método analítico Westergaard 1948. Las Figs. 2, 3, 4 y 5 muestran los elementos finitos que emplea el software Davids et al. [16]. El EverFE 2.25 trabaja con mallados de distintos grados de refinamiento y se compone de seis interfaces que realizan diferentes funciones: a) Geometry, contiene una herramienta CAD que permite construir un modelo geométrico e introducir los datos característicos de las capas y definir las fronteras del dominio del modelo. b) Material, almacena los parámetros de rigidez de cada material, densidades, coeficiente de expansión térmica del concreto hidráulico y las condiciones de liga entre capas. c) Loading, almacena los parámetros 
de carga, tipo de eje, lado y ancho del contacto de rueda (área de contacto rectangular), ubicación sobre el plano x-y de la(s) losa(s) y carga por temperatura. d) Dowel, contiene los parámetros de rigidez y geometría de las dovelas y barras de amarre, además, condiciones de liga entre dovelas y el concreto hidráulico de la(s) losa(s). e) Interlock, almacena abertura de las juntas con su respectiva rigidez, acompañado de la opción de modelo lineal a no lineal. f) Meshing, determina los parámetros de mallado. g) Solver, ejecuta el solucionador de elementos finitos. h) Results, permite obtener los resultados del análisis. Los pasos típicos del procedimiento de análisis de un problema con EverFE 2.25, son: ingresar los datos de dimensionamiento, número de capas, condiciones de frontera, espesores y parámetros de rigidez (módulos elásticos, reacción y de Poisson), otros factores inherentes al material (densidades y coeficiente de expansión térmica del concreto hidráulico), carga, ancho y largo del área de contacto (el programa trabaja con área de contacto rectangular) espaciamiento de las llantas, carga por temperatura, dovelas y barras de amarre con sus propiedades geométricas, de rigidez y liga con el concreto hidráulico, rigidez y abertura de las juntas, elegir combinación de mallado, ejecutar el solucionador y obtener resultados Davids [17]-[19].

Fig. 2. ELEMENTO FINITO BRICK CON 20 NODOS
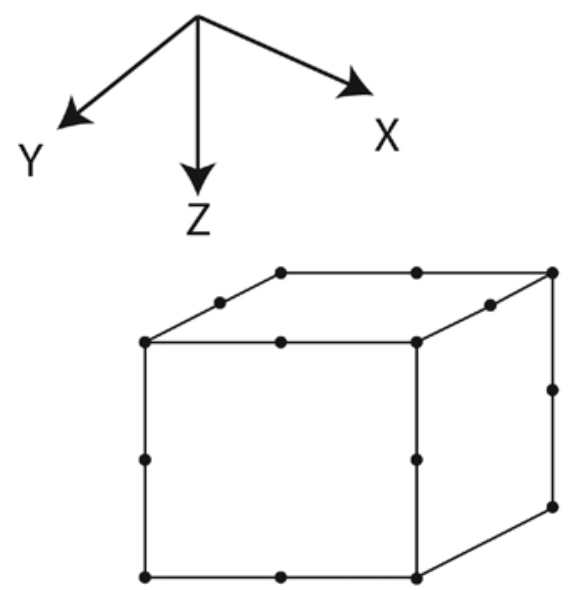

Fuente: [15].
Fig. 3. ELEMENTO FINITO INTERFACE CON 16 NODOS

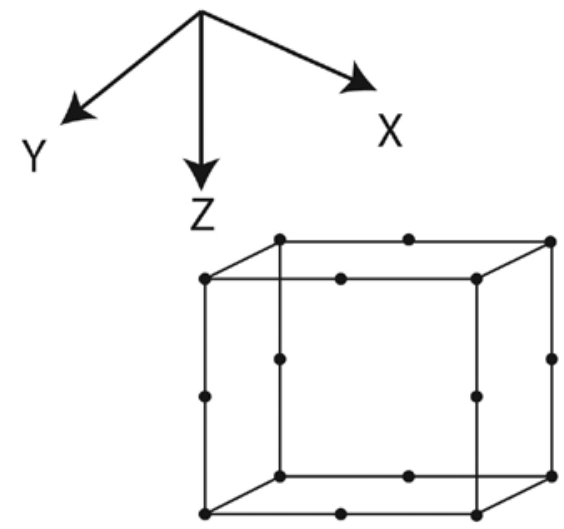

Fuente: [15]

Fig. 4. ELEMENTO FINITO DENSE LIQUID CON 8 NODOS

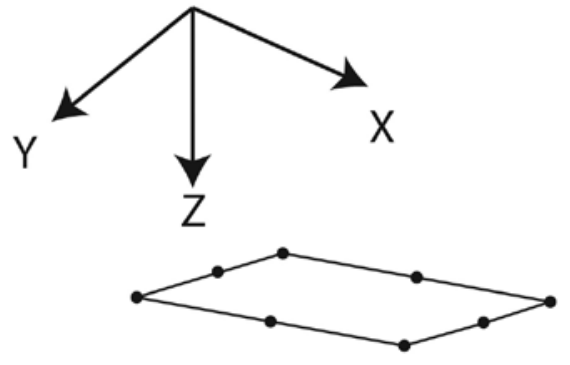

Fuente: [15]

Fig. 5. ELEMENTO FINITO BEAM CON 2 NODOS

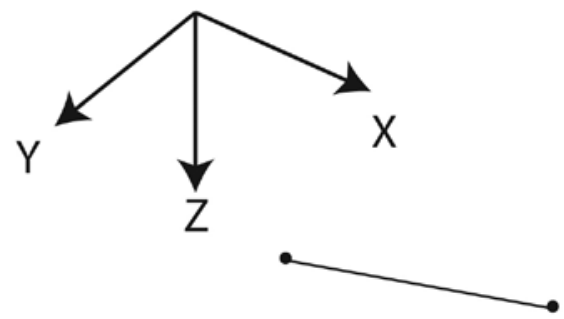

Fuente: [15]

\subsection{1 Área de contacto}

Como el método analítico Westergaard 1948 maneja en su formulación un área de contacto circular, diferente al presentado por el modelador tridimensional elástico EverFE 2.25, se calcula en las 
expresiones (6) y (7) la relación entre las áreas de contacto circular y rectangular. La Fig. 6 justifica la procedencia del nuevo dato de cálculo. Un estudio de los efectos de otros tipos de distribuciones de carga se trata en la referencia de los autores [20].

$$
\begin{gathered}
A_{\text {circular }}=\pi \mathrm{a}^{2}=(\pi)(6 \mathrm{in})^{2}=113.097 \mathrm{in}^{2} \\
\mathrm{~A}_{\text {rectangular }}=113.097 \mathrm{in}^{2}=\left(\mathrm{L}^{2}\right) \Rightarrow \\
\mathrm{L}=10.634 \mathrm{in} \approx 270 \mathrm{~mm} \quad(7)
\end{gathered}
$$

Donde $L$ es el ancho del área de contacto rectangular, $\mathrm{A}$ el área de contacto y a el radio del área circular.

Fig. 6. ÁREAS DE CONTACTO CIRCULAR Y RECTANGULAR

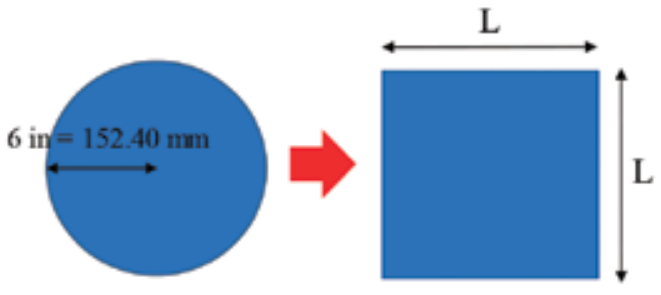

Fuente: Ios autores.

\subsection{Funciones de transferencia PCA}

Se aplicaron las funciones de transferencia de la Portland Cement Association - PCA Packard y Tayabji [21], calculando el número de repeticiones de carga permitidas con las expresiones (8), (9) y (10).

$$
\begin{gathered}
N_{f}=10^{11.737-12.077\left(\frac{\sigma}{S c}\right)} \operatorname{para} \frac{\sigma}{S c} \geq 0.55 \\
N_{f}=\left(\frac{4.2577}{\frac{\sigma}{S c}-0.4325}\right)^{3.268} \\
\text { para } 0.45<\frac{\sigma}{S c}<0.55 \quad(9) \\
N_{f}=\text { Sin limite para } \frac{\sigma}{S c} \leq 0.45
\end{gathered}
$$

Donde $\mathrm{N}_{\mathrm{f}}$ número de repeticiones de carga permitidos, $\sigma$ esfuerzo de tensión aplicado, Sc módulo de rotura del concreto y $\sigma / S c$ Relación de esfuerzo.

\section{RESULTADOS}

En la Tabla IV se presentan los resultados del método analítico Westergaard 1948.
TABLA IV

RESULTADOS DE CONTROL DE DISEÑO ESTRUCTURAL POR FATIGA DE UN PAVIMENTO RÍGIDO DE LOSAS JCPC

\begin{tabular}{|l|c|c|}
\hline \multicolumn{1}{|c|}{ Ubicación carga } & Parámetro & Analítico \\
\hline Interior & $\sigma_{\mathrm{A}}(\mathrm{Mpa})$ & 1.05 \\
\hline Esquina (Corner) & $\sigma_{\mathrm{F}}(\mathrm{Mpa})$ & 1.35 \\
\hline Borde (Edge) & $\sigma_{\mathrm{B}}(\mathrm{Mpa})$ & 1.99 \\
\hline
\end{tabular}

Fuente: los autores.

Se observa que el máximo esfuerzo principal a tensión está ubicado en el borde de la losa. Lo cual conlleva tomar la decisión de dimensionar las losas JCPC para pavimentos con solicitación de carga en el borde.

La tabla $\mathrm{V}$ contiene los resultados con solicitación de carga en el borde del método analítico Westergaard 1948 - utilizado como validador - y el modelo de elementos finitos EverFE 2.25.

TABLA V

VALIDACIÓN DE LOS RESULTADOS DE CONTROL DE DISEÑO ESTRUCTURAL DE UN PAVIMENTO RÍGIDO DE LOSAS JCPC

\begin{tabular}{|c|c|c|c|c|}
\hline $\begin{array}{c}\text { Ubicación } \\
\text { carga }\end{array}$ & Parámetro & Analítico & $\begin{array}{c}\text { Elementos } \\
\text { Finitos 3D }\end{array}$ & \%Variación \\
\hline $\begin{array}{c}\text { Borde } \\
\text { (Edge) }\end{array}$ & $\sigma_{\mathrm{B}}(\mathrm{Mpa})$ & 1.99 & 1.88 & $5.5 \%$ \\
\hline
\end{tabular}

Fuente: los autores.

Se observa que los porcentajes de variación del modelo numérico frente al analítico son inferiores al $5.5 \%$ lo cual permite concluir que las decisiones tomadas en la modelación con EverFE 2.25 son adecuadas para implementar los modelos que introduce base granular y un modelo más robusto de losas JCPC. En la Fig. 7 se presenta el resultado del modelo de elementos finitos EverFE 2.25.

Fig. 7. MÁXIMOS ESFUERZOS PRINCIPALES EN EL PLANO X-Y
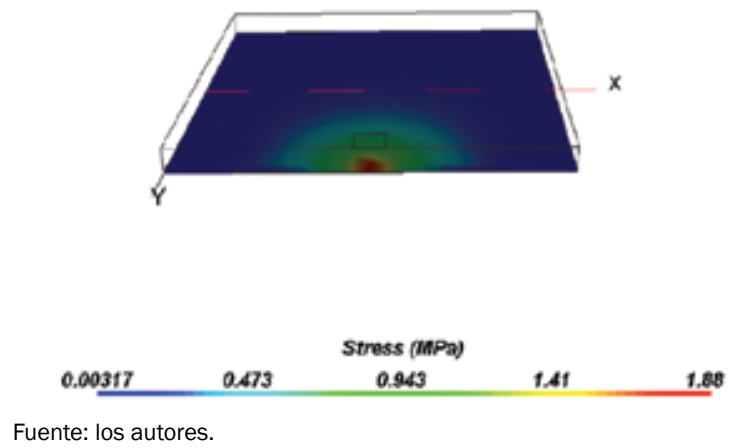

La Fig. 7 muestra el resultado gráfico de esfuerzos, correspondiente al parámetro de diseño empírico-mecanístico por medio de isocontornos. En 
la Fig. 7 se observa que los máximos esfuerzos se concentran bajo la llanta y en las fibras inferiores de la losa tal como lo sugiere el método analítico Westergaard 1948.

\subsection{Modelo de losas JCPC para carreteras de dos carriles}

Se desarrollaron dos modelos para analizar la respuesta de esfuerzos de la estructura de pavimento, conformado por tres losas cuadradas por carril en serie, siendo cada una de $3.5 m * 3.5 m$ de dimensión en planta y abertura de juntas de $5 \mathrm{~mm}$ de espesor, dimensiones características para anticipar las fallas por contracción y alabeo del concreto hidráulico. Se evaluó con y sin peso propio la estructura, para contemplar factores que lleven a tener análisis más realistas.

\subsubsection{Sin peso propio de la estructura}

La Fig. 8 presenta el desarrollo del esfuerzo en el plano X-Y de las fibras inferiores de las losas JCPC para pavimento de carretera de dos carriles sin peso propio. Bajo la llanta se concentra el máximo esfuerzo principal $\sigma_{B}=1.89 \mathrm{Mpa}$.

Fig. 8. ESFUERZOS EN EL PLANO X-Y DEL PAVIMENTO DE CARRETERA DE DOS CARRILES SIN PESO PROPIO
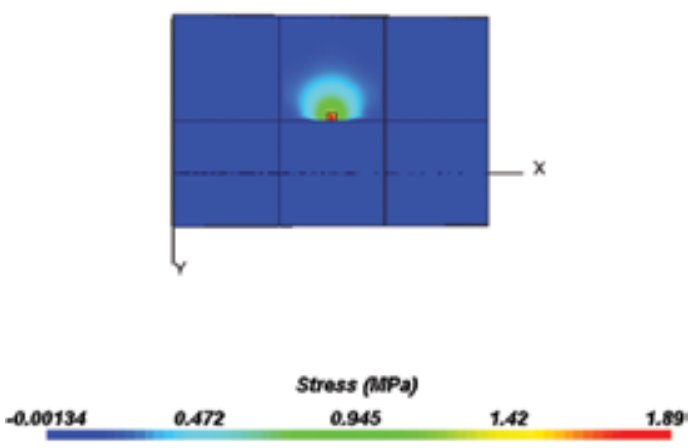

Fuente: los autores.

\subsubsection{Con peso propio de la estructura}

La Fig. 9 presenta el desarrollo del esfuerzo en el plano $X-Y$ de las fibras inferiores de las losas JCPC para pavimento de carretera de dos carriles con peso propio. Peso que se activa mediante el parámetro gravimétrico densidad, siendo para el concreto hidráulico $2200 \mathrm{~kg} / \mathrm{m}^{3}$ y la base granular $2100 \mathrm{~kg} / \mathrm{m}^{3}$. Bajo la llanta se concentra el máximo esfuerzo principal $\sigma_{B}=1.86 \mathrm{Mpa}$.
Fig. 9. ESFUERZOS EN EL PLANO X-Y DEL PAVIMENTO DE CARRETERA DE DOS CARRILES CON PESO PROPIO
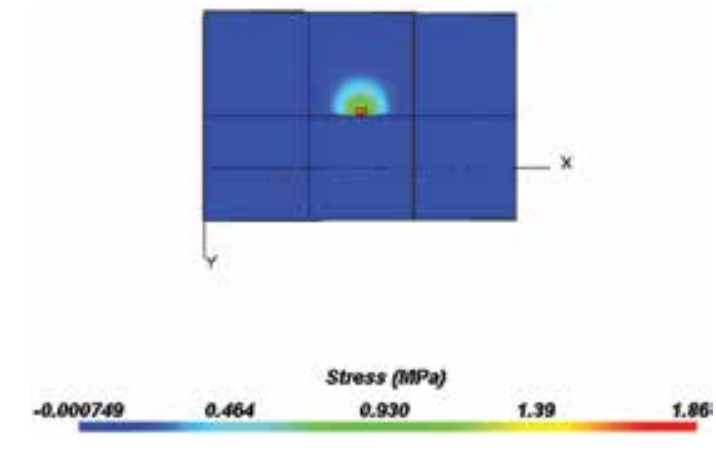

Fuente: los autores.

\subsubsection{Resumen de resultados con y sin peso propio de la estructura}

La Tabla VI contiene el resumen de resultados con y sin peso propio de la estructura, determinando porcentajes de variación poco significativos.

TABLA VI

VARIACIÓN DE LOS RESULTADOS DE CONTROL DE DISEÑO ESTRUCTURAL DE UN PAVIMENTO RÍGIDO DE LOSAS JCPC CON Y SIN PESO PROPIO DE LA ESTRUCTURA

\begin{tabular}{|c|c|c|c|c|}
\hline $\begin{array}{c}\text { Ubicación } \\
\text { carga }\end{array}$ & Parámetro & $\begin{array}{c}\text { Sin peso } \\
\text { propio }\end{array}$ & $\begin{array}{c}\text { Con peso } \\
\text { propio }\end{array}$ & \%Variación \\
\hline $\begin{array}{c}\text { Borde } \\
\text { (Edge) }\end{array}$ & $\sigma_{\mathrm{B}}(\mathrm{Mpa})$ & 1.89 & 1.86 & $1.6 \%$ \\
\hline
\end{tabular}

Fuente: los autores.

\subsubsection{Número de repeticiones de carga permitidos}

Para la estructura de pavimento rígido de losas JCPC aplicado para carreteras de dos carriles estudiado hasta el momento, podría soportar 25.253,697 repeticiones de carga, valor calculado con un esfuerzo de tensión de 1.89 Mpa, módulo de rotura de $4 \mathrm{Mpa}$ y relación de esfuerzo de 0.46 .

\section{CONCLUSIONES}

Los resultados obtenidos del modelo de elementos finitos desarrollado con el software libre EverFE 2.25 fueron satisfactoriamente validados con el método analítico Westergaard 1948, lo cual permite analizar y dimensionar losas JCPC para carreteras de dos carriles.

Se evidenció que no es necesario contemplar el análisis y dimensionamiento con peso propio de 
la estructura, porque el porcentaje de variación es poco significativo. Por lo tanto, se recomienda omitir el parámetro densidad sobre el análisis y así generar un coste computacional menor.

El método de los elementos finitos ha demostrado ser muy eficaz y en el caso particular, permitió analizar y dimensionar losas JCPC para carreteras de dos carriles, por lo tanto, marca un camino por seguir para estudiar la sensibilidad de distintos factores que pueden afectar el diseño de pavimentos rígidos.

Generado el interés de profundizar en la aplicabilidad del método de los elementos finitos en el análisis y dimensionamiento de losas JCPC para carreteras de dos carriles, en próximos estudios se analizarán condiciones de carga de distintos vehículos y los comportamientos producidos por la presencia de dovelas y barras de amarre en juntas transversales y longitudinales. De tal manera que se pueda generar análisis y dimensionamiento más realista.

\section{REFERENCIAS}

[1] A. M. Ioannides, Pavement Fatigue Concepts: A Historical View. Proc., 6th International Conference on Concrete Pavement Design and Materials for High Performance, vol. 3, Purdue University, West Lafayette, Ind., 1997.

[2] P. A. Okamoto, "Report on Review of Concrete Fatigue Models", PCA R\&D Serial No. 2213. Portland Cement Association, Skokie, III, 1999.

[3] L. Titus-Glover, J. Mallela, M. Darter, G. Voigt and S. Waalkes, Enhanced Portland Cement Concrete Fatigue Model for StreetPave. Transportation Research Record: Journal of the Transportation Research Board, vol. 1919, pp. 29-37, Jan, 2005.

[4] J.H. Jeong and D. Zollinger, Development of Test Methodology and Model for Evaluation of Curing Effectiveness in Concrete Pavement Construction. Transportation Research Record: Journal of the Transportation Research Board, vol. 1861, pp. 17-25, 2003.

[5] R. Mallick and El-Korchi, Pavement engineering: principles and practice. United States of American, CRC Press Taylor \& Francis Group., 2013, pp. 48-49.
[6] R. Mallick and El-Korchi. Pavement engineering: principles and practice. United States of American, CRC Press Taylor \& Francis Group., 2009, pp. 40-41.

[7] ASTM, Standard Test Method for Nonrepetitive Static Plate Load Tests of Soils and Flexible Pavement Components, for Use in Evaluation and Design of Airport and Highway Pavements, American Society for Testing and Materials, United States of American, 2016.

[8] Ping, Z. Yang, and Z. Gao, “Field and Laboratory Determination of Granular Subgrade Moduli". ASCE Journal of Performance of Constructed Facilities, vol. 16, No. 4, pp. 149-159, 2002.

[9] AASHTO, Standard Method of Test for Determining the Resilient Modulus of Soils and Aggregate Materials. American Association of State Highway and Transportation Officials, United States of American, 2012.

[10] S. H. Joh, M. R. Cho, T. H. Kang, S. Kwon and J. Nam, "Pavement Integrity Scanner to Characterize Modulus Contrast Between Near-Surface and Deeper Material in Concrete Pavements". Transportation Research Record: Journal of the Transportation Research Board, vol. 2070, pp. 39-48, 2008.

[11] ASTM, Standard Test Method for Static Modulus of Elasticity and Poisson's Ratio of Concrete in Compression. American Society for Testing and Materials, United States of American, 2014.

[12] Y. Huang, Pavement analysis and desing. Kentucky United States of American, Prentice hall, 2004.

[13] LCPC, Conception et dimensionnement des structures de chaussée, Guide technique. LCPC and SETRA, Paris, 1994, pp. 163.

[14] A. Papagiannakis and E. Masad, Pavement design and materials. United States of American, John Wiley \& Sons, 2008, pp. 221-225.

[15] J. N. Reddy, Mechanics of Laminated Composite Plates and Shells Theory and Analysis, 2nd ed. CRC Press, Boca Raton, Fla, 2004.

[16] W. G. Davids, Z. M. Wang, G. Turkiyyah, J. Mahoney and D. Bush, "Finite Element Analysis of Jointed Plain Concrete Pavement with EVERFE 2.2." Transportation Research Record 1853: Journal of the Transportation Research Board, TRB, National Research Council, United States of American, pp. 92-99, 2003. 
[17] W. G. Davids, Foundation Modeling for Jointed Concrete Pavements. Transportation Research Record: Journal of the Transportation Research Board, vol. 1730, pp. 34-42, 2000.

[18] W. G. Davids, EverFE Theory Manual. United States of American, University of Maine Dept. of Civil and Environmental Engineering, 2003.

[19] W. G. Davids, G. M. Turkiyyah and J. P. Mahoney, “EverFE: Rigid Pavement Three-Dimensional Finite Element Analysis Tool". In Transportation Research Record 1629, TRB, National Research Council, United States of American, 1998, pp. 41-49.
[20] M. R. Pallares and J.A. Pulecio, "Effect of a dual tire pressure on the design parameters of thick asphalt pavements using finite element freeware," Dyna, No. 196, pp. 194-203, Apr. 2016.

[21] R.G. Packard and S.D. "Tayabji. New PCA thickness design procedure for concrete highway and street pavements", The Third International Conference on Concrete Pavement Design and Rehabilitation, Purdue University, West Lafayette, United States of American, 1985. 